\title{
Precise Time-of-Flight Calculation For 3-D Synthetic Aperture Focusing
}

\section{Andresen, Henrik; Nikolov, Svetoslav; Jensen, Jørgen Arendt}

Published in:

I E E E Transactions on Ultrasonics, Ferroelectrics and Frequency Control

Link to article, DOI:

10.1109/TUFFC.2009.1264

Publication date:

2009

Document Version

Publisher's PDF, also known as Version of record

Link back to DTU Orbit

Citation (APA):

Andresen, H., Nikolov, S., \& Jensen, J. A. (2009). Precise Time-of-Flight Calculation For 3-D Synthetic Aperture Focusing. I E E E Transactions on Ultrasonics, Ferroelectrics and Frequency Control, 56(9), 1880-1887. https://doi.org/10.1109/TUFFC.2009.1264

\section{General rights}

Copyright and moral rights for the publications made accessible in the public portal are retained by the authors and/or other copyright owners and it is a condition of accessing publications that users recognise and abide by the legal requirements associated with these rights.

- Users may download and print one copy of any publication from the public portal for the purpose of private study or research.

- You may not further distribute the material or use it for any profit-making activity or commercial gain

- You may freely distribute the URL identifying the publication in the public portal 


\title{
Precise Time-of-Flight Calculation For 3-D Synthetic Aperture Focusing
}

\author{
Henrik Andresen, Svetoslav Ivanov Nikolov, and Jørgen Arendt Jensen
}

\begin{abstract}
Conventional linear arrays can be used for 3-D ultrasound imaging by moving the array in the elevation direction and stacking the planes in a volume. The point-spread function is larger in the elevation plane, because the aperture is smaller and has a fixed elevation focus. Resolution improvements in elevation can be achieved by applying synthetic aperture focusing to the beamformed-in-plane RF data. The proposed method uses a virtual source placed at the elevation focus for postbeamforming. This has previously been done in 2 steps, in-plane focusing followed by synthetic aperture postfocusing in elevation, due to lack of a simple expression for the exact time of flight. This paper presents a new single step method for calculating the time of flight for a 3-D case using a linear array. The new method is more flexible and is able to beamform a fewer number of points much more efficiently. The method is evaluated using both simulated data and phantom measurements using the RASMUS experimental scanner. Computational cost of the method is higher than the 2-step method for a full volume beamforming, but it allows for a reduction of an order-of-magnitude if 3 planes are used for real-time visualization. In addition, the need for a temporary storage of beamformed data is removed.
\end{abstract}

\section{INTRODUCTION}

$\mathrm{I}^{\mathrm{N}}$ $\mathrm{N}$ ultrasound, 3-D volume acquisition has long been desired for several reasons. A full volume of an organ or vascular section can help visualize defects and ailments. More important is the ability to reduce operator dependence on the ultrasound scan. For 2-D ultrasound scannings, only a single slice is visible, and the exact outcome is largely dependent on the physician being able to control the orientation of the scan-plane. With a 3-D volume, the scan-plane can be changed by the physician after the scan is complete, allowing for a better diagnosis that reduces the necessity of additional examinations of patients.

Two basic ways to acquire $3-\mathrm{D}$ images exist: using a 2-D array [1] or a mechanically moved 1-D array. The use of linear array transducers to acquire 3-D volume ultrasound (US) images is more common than 2-D array acquisition. A linear array is readily commercially available, has relatively few elements, and can be used with modern ultrasound scanners. A drawback is that the array must be mechanically moved for a volume acquisition, in-

Manuscript received August 19, 2008; accepted March 23, 2009. This work was supported by grant 71122 from the Danish Ministry of Science, Technology and Innovation, grant 9700883, 9700563, and 26-040024 from the Danish Science Foundation, and by B-K Medical Aps, Denmark.

H. Andresen and J. A. Jensen are with the Center for Fast Ultrasound Imaging, DTU Elektro, Technical University of Denmark, Lyngby, Denmark.

H. Andresen and S. I. Nikolov are with B-K Medical, Herlev, Denmark.

Digital Object Identifier 10.1109/TUFFC.2009.1264 creasing acquisition time. With linear arrays, 3-D volume acquisition has traditionally been done by imaging one plane and moving the transducer, creating the 3-D volume by stacking individual planes. These volumes have good lateral and depth resolution, but poor elevation resolution and contrast because of the small elevation aperture size. Another drawback is increased acquisition time due to the large increase in scan-lines. Possible solutions are proposed by [2], [3].

Using a synthetic aperture ( $\mathrm{SA}$ ) focusing technique to improve the elevation resolution of a fixed focus transducer has been shown feasible in [4]. This has further been used in [5]-[7] with linear and phased-array transducers, to allow for both lateral and elevation focusing. These works have shown a significant increase in elevation resolution, contrast, and signal-to-noise ratio (SNR) when applying a 2-step elevation beamforming method. The 2-step method assumes that the individual emissions for creating the high-resolution image planes can be combined into a single plane. If the transducer is continually moving, a new plane must be beamformed for each emission, increasing the cost of creating the intermediate data and the cost of postbeamforming. The initial in-plane beamforming can be performed by either conventional B-mode beamforming or SA focusing, where SA focusing has been used for a variety of different approaches [8]-[13].

The method presented in this paper calculates the time of flight (ToF) in a single step for a given point in $3-\mathrm{D}$ space, using a moving linear array with a fixed elevation focus. This removes the need for doing the beamforming in 2 steps, which has several advantages. The beamforming does not rely on an intermediate data set, which for moving transducers can become very large, and only requires a single interpolation of data. The method is fully scalable with the final number of imaging points, allowing a full 3-D focusing for a single plane to be calculated very effectively. The 2-step method is largely invariant in processing requirements regardless of the desired number of beamformed points.

Section II describes the equations and theory used in the ToF-calculation, and Section III considers the processing requirement for different beamforming options. The measurement setup is described in Section IV, and Section $\mathrm{V}$ shows the results from both simulation and measurements. The paper concludes in Section VI.

\section{Time-OF-Flight Calculation}

SA focusing applied to linear arrays is traditionally done by calculating the ToF for a spherical wave emanating 


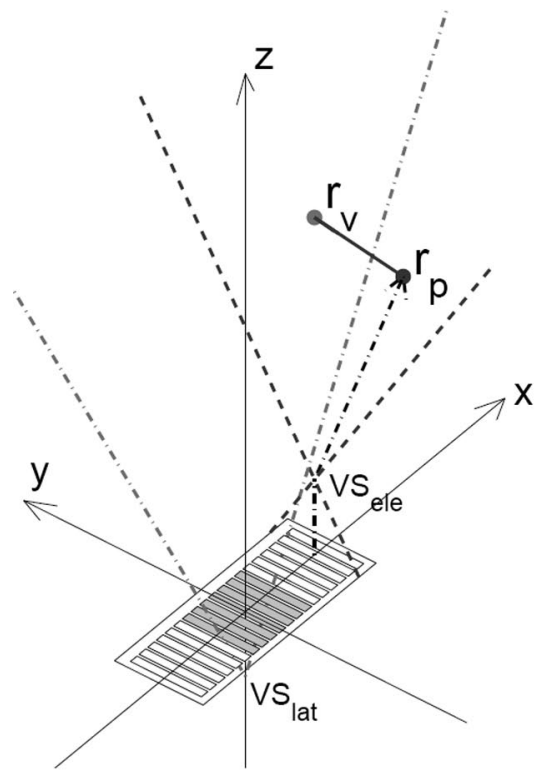

(a)

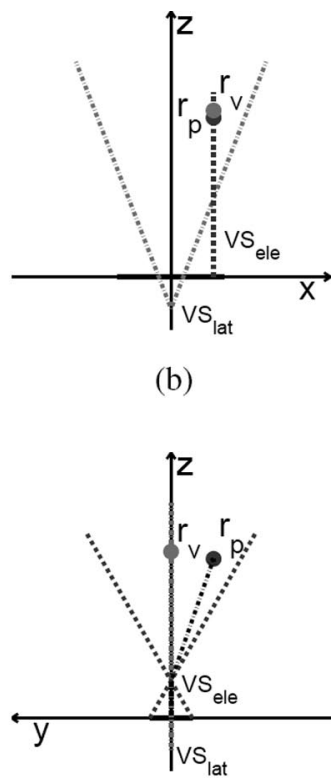

(c)

Fig. 1. (a) 3-D illustration of the initial part of the ToF calculation. The dotted lines are the lateral VS acceptance angle. The point $\vec{r}_{p}$ is the desired beamformed point. The dashed lines represent the acceptance angle of the elevation VS placed at the elevation focus. A virtual point $\vec{r}_{v}$ is created in the XZ plane. The point is placed so the wavefront will reach both $\vec{r}_{p}$ and $\vec{r}_{v}$ at the same time; (b) and (c) are projections in the XZ and $\mathrm{YZ}$ plane, respectively.

from a single point, allowing each emission to contribute to the focusing of the entire insonified region. Because SA has an inherently low SNR, virtual sources (VS) [14]-[17] and frequency modulation [18] are used to increase the emitted energy. For 2-D images, a simple geometric model with a transmitting element, a point of interest, and a set of receive elements are used to generate a low-resolution image. The transmitted wave has a propagation in the elevation plane dependent on the geometry of the physical elements. This results in information of a small volume to be contained in the received pulse-echo signal. For 2-D imaging, the contribution from the elevation plane slice is effectively averaged, giving a poor elevation resolution compared with lateral and axial. By moving the linear array, a monostatic sampling of a larger array in the elevation direction is acquired.

Previous methods use first in-plane beamforming, where the beamformed lines have to be sampled according to the Nyquist criterion. The method presented here calculates the direct ToF for a given 3-D point. Points outside the acceptance angles of the virtual sources are not valid and will be discarded. In the description and in figures, $x, y$, and $z$ are used to denote lateral, elevation, and axial directions, respectively.

The method of calculating the ToF uses 2 virtual sources, one in the lateral and one in the elevation direction. The lateral VS, denoted $\mathrm{VS}_{\text {lat }}$, is placed at the transmit VS used to increase SNR in SA focusing, and the elevation $\mathrm{VS}$, denoted $\mathrm{VS}_{\text {ele }}$, is placed at the elevation focus depth of the transducer and used to increase elevation resolu-

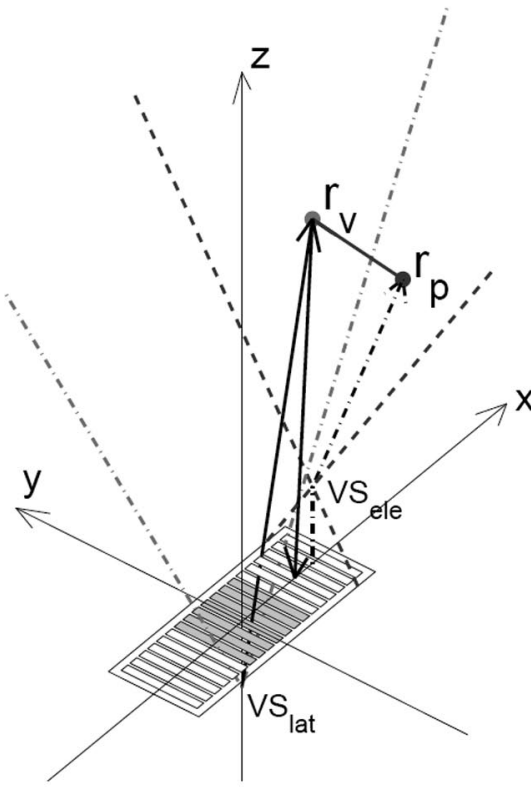

(a)

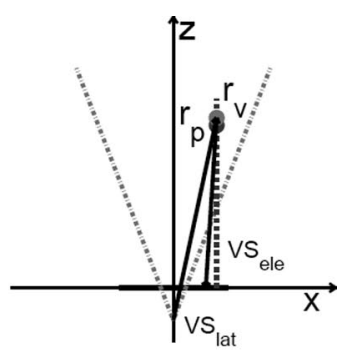

(b)

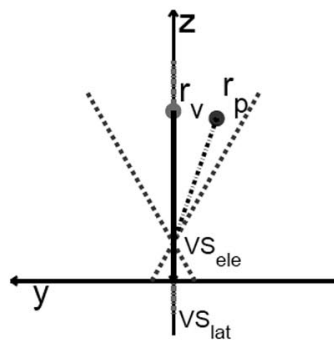

(c)
Fig. 2. (a) 3-D illustration of the final step of the ToF calculation. To calculate the ToF for each receive channel, normal 2-D SA focusing is used for the point $\vec{r}_{v}$. The solid line represents an example of a single transmit-receive path used for a receive channel; (b) and (c) are projections in the XZ and YZ plane, respectively.

tion. The process is shown stepwise in Figs. 1 and 2. The desired beamformed point is denoted $\vec{r}_{p}$, the dotted lines show the acceptance angle for $\mathrm{VS}_{\text {lat }}$, and the dashed lines show the acceptance angle for $\mathrm{VS}_{\text {ele. }}$ The first part of the method projects the point $\vec{r}_{p}$ onto the $x z$-plane (lateraldepth) by letting the depth of the point $\vec{r}_{v}$ equal the distance traveled by the sound from the transducer to $\mathrm{VS}_{\text {ele }}$ and to $\vec{r}_{p}$, on the $y z$-plane. This is calculated by placing $\mathrm{VS}_{\text {ele }}$ on the same lateral position as $\vec{r}_{p}$. The depth of the new point will be given by

$$
\begin{aligned}
z_{\text {proj }}= & \sqrt{r_{p, y}^{2}+\left(r_{p, z}-\mathrm{VS}_{\text {ele }, z}\right)^{2}} \cdot \operatorname{sign}\left(r_{p, z}-\mathrm{VS}_{\text {ele }, z}\right) \\
& +\mathrm{VS}_{\text {ele }, z},
\end{aligned}
$$

where $r_{p, y}, r_{p, z}$ is the elevation and depth position of $\vec{r}_{p}$ relative to the transducer and $\mathrm{VS}_{\text {ele, } z}$ is the depth of the elevation VS. This step is visualized in Fig. 1. The virtual point, denoted $\vec{r}_{v}$, will have the coordinates $\left(r_{p, x}, 0, z_{\text {proj }}\right)$. This virtual point is used for the ToF calculation using in-plane SA focusing. The equation for the total ToF for a transmission to the $m$ th receive element is given by

$$
t_{\mathrm{ToF}, m}=\frac{\left|\vec{r}_{v}-\mathrm{VS}_{\mathrm{lat}}\right|+\left|\vec{r}_{v}-\vec{r}_{\mathrm{rcv}, m}\right|}{c},
$$

where $\mathrm{VS}_{\text {lat }}$ is the position of the transmit VS, $\vec{r}_{\mathrm{rcv}, m}$ is the position of the $m$ th receiving element, and $c$ is the speed of sound. The path is shown by the solid line in Fig. 2. The signal amplitude for a single point is given by summing the received signals at the time instances calculated by (2), which yields 


$$
s\left(\vec{r}_{p}\right)=\sum_{j=1}^{J} \sum_{m=1}^{M} \sum_{n=1}^{N} a_{j, m, n} \cdot g_{j, m, n}\left(t_{\mathrm{ToF}, m}\right)
$$

where $a_{j, m, n}$ is the apodization, and $g_{j, m, n}$ is the signal for the $m$ th receive channel of the $n$th emission at the $j$ th elevation position, $M$ is the number of receive elements, $N$ is the number of transmit $\mathrm{VS}$, and $J$ is the number of elevation positions.

\section{Performance Considerations}

A main difference between the previous 2-step approach and the proposed method is how many beamformed points are required and the amount of temporary data needed. A volume is often visualized in real-time using a single plane or 3 orthogonal planes. This will allow the proposed method to save processing power, because fewer resulting points are required. The 2-step approach is largely dependent on the amount of data sampled, with an increase in beamforming requirements for an increased resolution and sampling depth. The required lateral and axial sampling density for the first step is calculated from [19]. The required lateral sampling is

$$
\delta \theta \leq \sin ^{-1}\left(\frac{\lambda}{\left(1+\frac{B_{\text {rel }}}{2}\right) D_{\text {eff }}}\right)
$$

where $\lambda$ is the wavelength, $B_{\text {rel }}$ is the $-6 \mathrm{~dB}$ relative bandwidth, and $D_{\text {eff }}$ is the size of the effective aperture, which is the convolution of the transmit and receive aperture [20]. The number of axially beamformed points is required at a minimum of

$$
N=\frac{4 d\left(1+\frac{B_{\text {rel }}}{2}\right)}{\lambda}
$$

where $d$ is the sampling depth. These calculations will have to be done for each image plane used for the second postbeamforming step. The second beamforming step is usually 2 orders of magnitude lower in beamforming calculations due to the monostatic nature, so this will be ignored in the considerations. Each beamformed point requires interpolation, which will degrade the signal or add increased computational complexity if a higher order interpolation scheme is used in this step. The amount of beamforming required in the first step is invariant in relation to the resulting volume size. In addition, the beamformed data have to be stored temporarily between the 2 steps, adding an additional requirement on bandwidth. The total bandwidth required is equal to the number of points in each image plane in the first step. The number of planes depends on whether the transducer is moving or stationary during each plane acquisition. For stationary transducers, the planes for a single elevation position can be summed before post-beamforming. If the transducer is moving, each emission must be beamformed individually
TABLE I. Required BeAmformed Points for 3-D SA Focusing and the Conventional 2-Step Beamforming.

\begin{tabular}{lcc}
\hline Beamforming method & Full volume & Single plane \\
\hline 2-step beamforming & $0.90 \cdot 10^{12}$ & $0.90 \cdot 10^{12}$ \\
3-D SA beamforming & $1.03 \cdot 10^{13}$ & $3.61 \cdot 10^{10}$ \\
\hline
\end{tabular}

in elevation. The total number of bytes to transfer for the stationary case is

$$
B_{\text {stat }}=N_{\text {axial }} \cdot N_{\text {lat }} \cdot N_{\text {ele }}
$$

where $N_{\text {axial }}$ is given by (5), $N_{\text {lat }}$ is calculated by the desired field of view (FOV), and (4), and $N_{\text {ele }}$ is the number of elevation positions. The bandwidth for the moving case is equal to

$$
B_{\mathrm{mov}}=B_{\mathrm{stat}} \cdot N_{\mathrm{emis}}
$$

where $N_{\text {emis }}$ is equal to the number of emissions per image plane.

The proposed method has a more elaborate beamforming scheme for each beamformed point, giving a larger number of points to be beamformed per emission. The total number of points can be reduced by applying an effective discretization to remove the points outside the elevation acceptance angle from the beamforming process. The discretization is trivial to calculate and negligible in the total computational cost.

For the beamforming of the images taken for this paper, a $7-\mathrm{MHz}$ transducer with a $60 \%$ relative bandwidth was used, and a scan depth of $140 \mathrm{~mm}$ is required for pulsecompression for the $120 \mathrm{~mm}$ view depth. The transducer has $\lambda$ pitch, resulting in a requirement of 260 scan-lines for a $90^{\circ}$-sector scan, and $\approx 2800$ samples axially. Table I shows the number of beamformed points required by both methods to perform elevation focusing for a full volume as well as for a single plane of the resulting volume. The numbers given are only the required beamformed points for the first step of the conventional method and do not contain any computational cost from interpolation or movement of data to a temporary storage.

This shows the new method has a higher computational cost for a full volume for the chosen setup, but it is able to beamform a single plane at a reduced cost. This will allow the method to be used advantageously if a visualization using, e.g., orthogonal planes is used. In addition to this, there is no need for a temporary storage. For the measured data, the temporary storage would be $438 \mathrm{MB}$ using (6), assuming 16-bit data. If the transducer was moving continuously during acquisition, the storage would have to be increased to $28 \mathrm{~GB}$ using (7), because the individual emissions will not be able to be summed due to the difference in elevation position.

\section{Measurement Setup}

All measurements were done with the RASMUS experimental scanner and a precision positioning system (called 
TABle II. Transducer and Measurement Parameters.

\begin{tabular}{lc}
\hline Transducer type & Linear array \\
\hline Number of receive elements & 64 \\
Center frequency, $f_{0}$ & $7.0 \mathrm{MHz}$ \\
Transducer element pitch & 0.208 \\
Transducer element height & $4.5 \mathrm{~mm}$ \\
Elevation focus & $25 \mathrm{~mm}$ \\
STA scan parameters & 7 \\
Elements in virtual source & 64 \\
Emissions for full STA & $-1 / 2$ \\
Lateral VS focusing F\# & $20 \mu \mathrm{s}$ \\
FM-chirp length & $80 \mathrm{~mm}$ \\
Scan depth & Blackman \\
Receive apodization & 2 \\
Lateral receive F\# & $0.09 \mathrm{~mm}$ \\
Elevation step-size & \\
\hline
\end{tabular}

the xyz-system), both available at the Center for Fast Ultrasound imaging (CFU). RASMUS is the abbreviation for the remotely accessible software configurable multichannel ultrasound sampling system, and it was designed as a very flexible US system capable of transmitting arbitrary waveforms and storage of raw single channel data. A more detailed description is found in [21].

The full measurements were performed sequentially using a full imaging sequence for each elevation position. Each imaging sequence comprises 64 emissions. Each emission uses 7 elements for a defocused VS emission and 64 receive channels. The VS is placed just below the physical elements, but at the same lateral positions. The transducer is moved $0.09 \mathrm{~mm}$, equal to $0.41 \lambda$ in the elevation directions between each image sequence for either 201 or 301 scan planes. The elevation movement is done using the xyz-system, which allows for a precise movement of the transducer, using a stepper motor with a step size of $5 \mu \mathrm{m}$. An increase in SNR is gained by using a nonlinear FM chirp, and pulse compression is attained with a timeinverted matched filter [22]-[25].

The transducer and in-plane scan sequence parameters are shown in Table II. The same scan sequence is used for simulation and scanning of a single point-spread-function (PSF) phantom and a cyst phantom. A total of 201 or 301 planes are acquired during a scan. After data acquisition, the data are beamformed offline according to (3).

\section{RESULTS}

The method is based on the ability to calculate an accurate ToF for an arbitrary scatterer within the acceptance angle of the transmit VS and the elevation focus. To evaluate this, a simulated pulse-echo response is compared with the value calculated by (2) for a single off-axis scatterer at a position of $(25,8,75) \mathrm{mm}$ relative to the transducer center. This result is assumed to be representative for the methods and will be used to show the ability of the new and old method to give an accurate expression for the
Measured signal and estimated ToF for the point at $(25.00,8.00,75.00) \mathrm{mm}$ Bias $=0.0904 \lambda, \sigma=0.0054 \lambda$

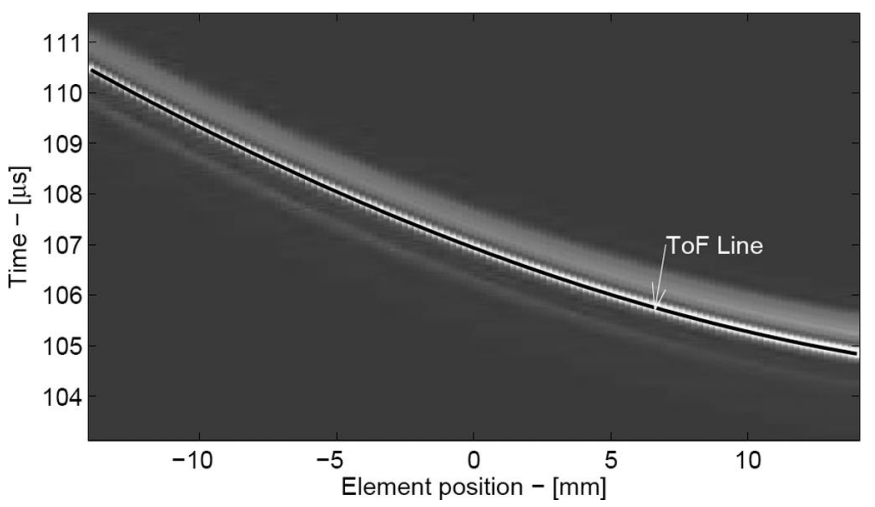

Fig. 3. Pulse-echo response from a single scatterer at position $(25,8,75) \mathrm{mm}$ relative to the transducer. The envelope of the RF-data is plotted at the receive channel position with the estimated ToF for each channel overlayed as a solid black line.

actual ToF. All simulations use Field II [26], [27] to calculate the pulse-echo response. Fig. 3 shows the envelope of the simulated pulse-echo response for an off-axis point scatterer, and the estimated ToF is shown by a solid black line. A bias and standard deviation (SD) is calculated for the difference between the ToF from (2) and the Field II simulations. The bias is calculated by

$$
e_{\text {bias }}\left(\vec{r}_{p}\right)=\frac{1}{N} \sum_{n=1}^{N} t_{f}\left(\vec{r}_{p}\right)-t_{e q}\left(\vec{r}_{p}\right)
$$

where $N$ is the number of receive channels, $t_{f}\left(\vec{r}_{p}\right)$ is the time simulated by Field II, and $t_{e q}\left(\vec{r}_{p}\right)$ is the time given by (2) calculated for the point $\vec{r}_{p}$. The time simulated in Field II is calculated at the peak of the envelope of the pulseecho signal. The $\mathrm{SD}$ is given by

$$
e_{\mathrm{SD}}\left(\vec{r}_{p}\right)=\sqrt{\frac{1}{N} \sum_{n=1}^{N}\left(t_{f}\left(\vec{r}_{p}\right)-t_{e q}\left(\vec{r}_{p}\right)\right)^{2}}
$$

The bias calculated from (8) for the proposed method is $0.090 \lambda$, and the $\mathrm{SD}$ is $0.005 \lambda$ for a $7 \mathrm{MHz}$ pulse, equal to a SD phase shift of $1.8^{\circ}$. For the conventional 2-step method for the same point, the bias is $0.12 \lambda$, and the SD is $0.0945 \lambda$ equal to a $34.0^{\circ}$ phase shift. The bias can be caused by the spatial extent and curvature of the array, because this is only zero at the exact focal point. In addition, the transmit VS will also introduce a small angledependent bias caused by the trailing wave. The reduction in SD indicates that the method is able to give a more accurate description of the ToF for an array.

The method's ability to synthesize a larger aperture in elevation is evaluated from the full-width at half max (FWHM) and the main-lobe to side-lobe energy ratio (MLSL) of both simulated and measured scatterers. The MLSL ratio is given by 


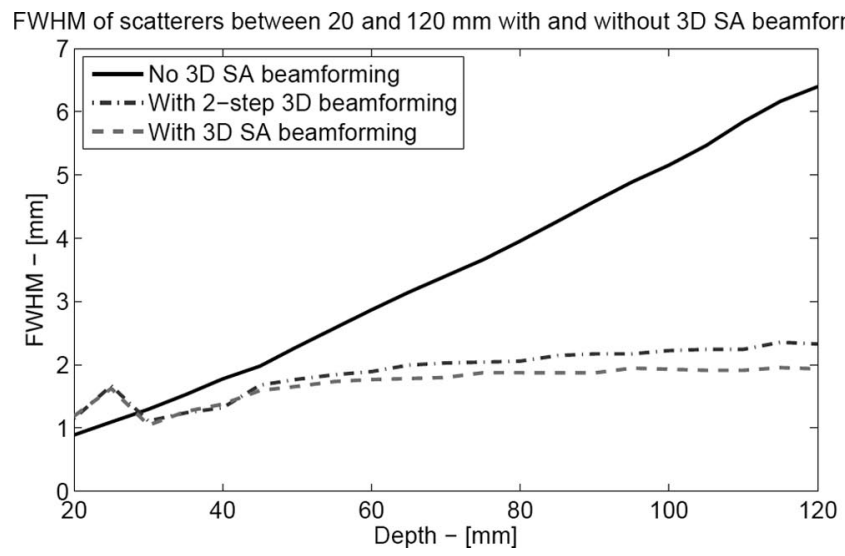

Fig. 4. Elevation full-width half $\max (\mathrm{FWHM})$ for scatterers between 20 and $120 \mathrm{~mm}$, with no, 2-step, and with 3-D focusing.

$$
\mathrm{MLSL}=10 \log _{10}\left(\frac{\sum_{n=n_{1}}^{n_{2}} s(n)^{2}}{\sum_{n=1}^{n_{1}-1} s(n)^{2}+\sum_{n=n_{2}+1}^{N} s(n)^{2}}\right),
$$

where $s(n)$ is the sampled PSF projected in the lateral direction, $n_{1}$, and $n_{2}$ is the index of the beginning and end of the main-lobe. The main-lobe is defined as the PSF above $-40 \mathrm{~dB}$. All PSFs are compared with the main-lobe of the elevation-focused PSF with the smallest elevation step size. This will avoid a poorer focusing giving a better MLSL ratio. A cyst phantom is used to measured the improvement of the contrast-to-noise ratio (CNR) in the lateral-depth plane, given by (11). The equation used is the same as given in [28]. For all measures, different stepping sizes in the elevation direction are investigated.

$$
\mathrm{CNR}=\frac{\left|\mu_{s}-\mu_{c}\right|}{\sqrt{0.5 \cdot\left(\sigma_{s}^{2}+\sigma_{c}^{2}\right)}}
$$

where $\mu_{s}$ and $\mu_{c}$ are the mean-value of the speckle and cyst area, respectively, and $\sigma_{s}^{2}$ and $\sigma_{c}^{2}$ are the variance of the speckle and cyst area.

\section{A. Simulated Scatterers}

In Fig. 4, the FWHM for scatterers at 20 to $120 \mathrm{~mm}$ of depths is shown. With no elevation focusing, a linear increase of the FWHM is seen as a function of depth. By applying 3-D focusing, the constant $\mathrm{F} \#$ allows for a near constant FWHM. The conventional 2-step focusing method shows results similar to the 3 -D focusing, but it has a slightly larger FWHM at deeper lying scatterers.

For different elevation stepping sizes, the effect on a simulated scatterer at $65 \mathrm{~mm}$ of depth is shown in Fig. 5. For a stepping size of $0.41 \lambda$ the side-lobes are below $-60 \mathrm{~dB}$, while for $2.05 \lambda$ stepping size they are below $-50 \mathrm{~dB}$. An increase of the elevation step size to $4.50 \lambda$ elevates the side-lobes to almost the same level as with no

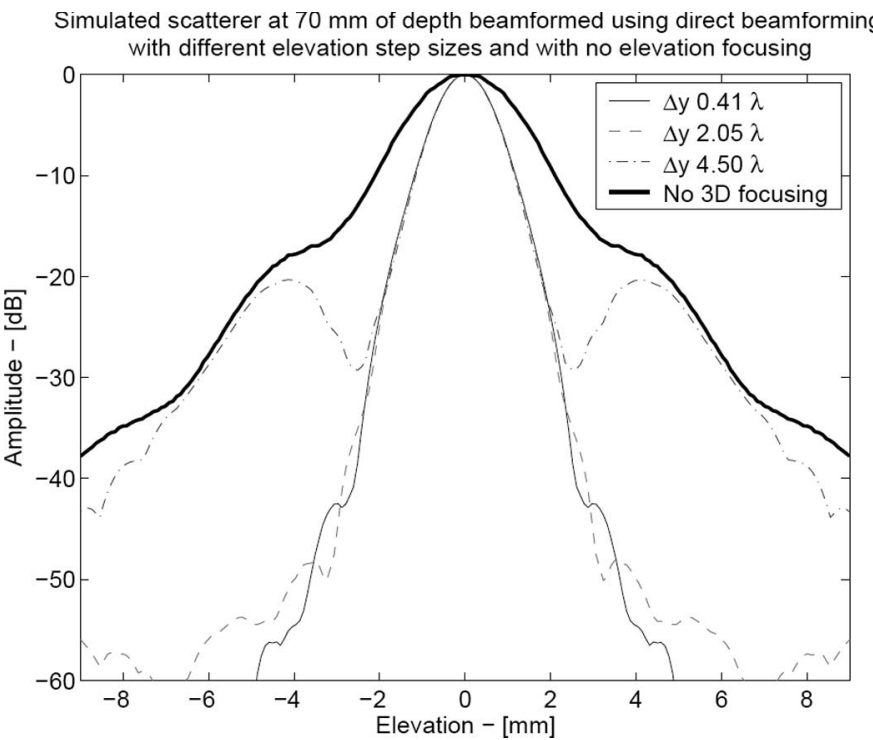

Fig. 5. Projection of the PSF of a simulated scatterer at $65 \mathrm{~mm}$ of depth for different elevation step sizes.

3-D focusing. The FWHM is unchanged by the stepping size, because the width of the synthesized aperture is the same. A comparison of the lateral FWHM has a difference of $0.21 \%$, which shows that no change is induced by the method.

The results of a larger analysis of the effect of different elevation step sizes are shown in Figs. 6 and 7. The FWHM is seen to increase very slowly when 3 -D focusing is applied. This FWHM is almost the same regardless of step size, which is in accordance with the theory, because this is defined mostly by the size of the effective aperture. The MLSL is almost identical for 0.5 to $2 \lambda$ with a slight increase for lower step sizes. Above $2 \lambda$, the side-lobes become an increasing problem, and the MLSL ratio is decreased.

\section{B. PSF Phantom}

The PSF phantom is created by embedding a small diamond within a block of agar. This was placed at approximately $65 \mathrm{~mm}$ of depth and measured stepwise as described in Section IV. The amplitude projected in the lateral plane of the PSF is shown in Fig. 8. The measurements show a higher average of the side-lobes compared with the simulation as well as an asymmetric side-lobe amplitude. The asymmetry can be caused by a slight angling of the transducer, or because the scatterer is not perfectly below the center elevation position. The improvement in FWHM and MLSL when applying 3-D SA focusing is better for simulation, but the starting point is also poorer. The values for the specific scatterer are given in Table III.

A measurement of the FWHM at $65 \mathrm{~mm}$ in both the lateral and elevation direction for simulations and measurements at $65 \mathrm{~mm}$ of depth is shown in Table III. The ratio with and without 3 -D focusing is 1.77 and 3.21 for 


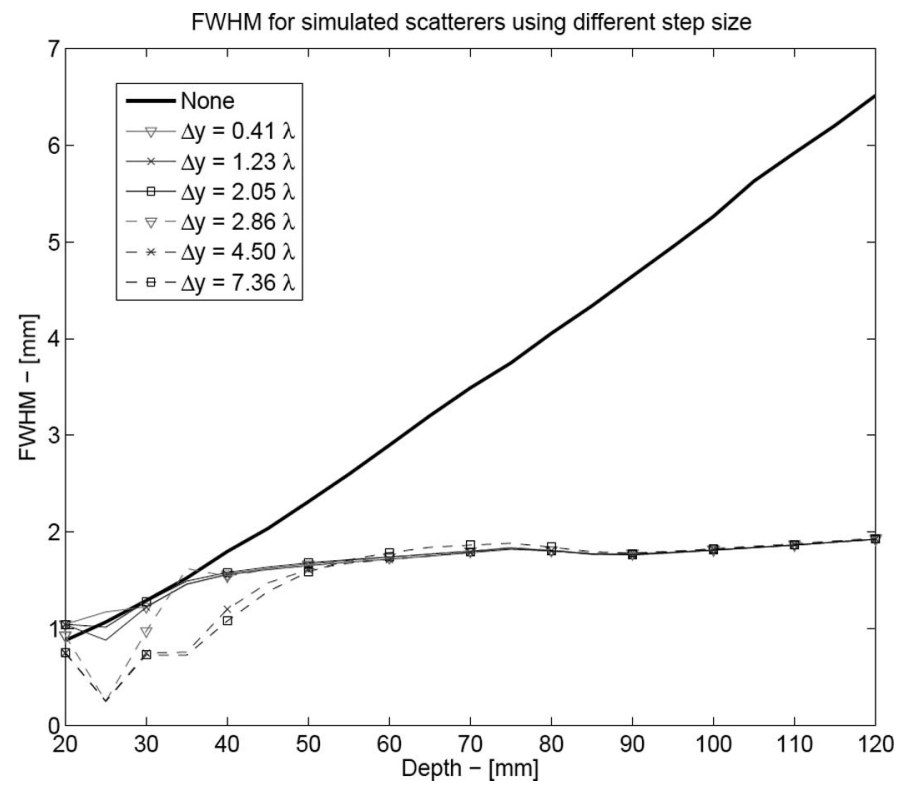

Fig. 6. Full-width half max (FWHM) for simulated scatterers between 20 and $120 \mathrm{~mm}$ of depth. Each line represents a different step size in elevation.

TABLE III. MLSL RATIO AND FWHM FOR SimULATED AND Measured PSF at a DePth OF 65 MM. ${ }^{1}$

\begin{tabular}{lcc}
\hline Type & $\begin{array}{c}\text { Simulation at } \\
65 \mathrm{~mm}\end{array}$ & $\begin{array}{c}\text { Phantom at } \\
65 \mathrm{~mm}\end{array}$ \\
\hline Elevation FWHM & $1.78 \mathrm{~mm}$ & $1.77 \mathrm{~mm}$ \\
0.09 mm step & $1.77 \mathrm{~mm}$ & $1.74 \mathrm{~mm}$ \\
0.45 mm step & $1.75 \mathrm{~mm}$ & $1.74 \mathrm{~mm}$ \\
0.99 mm step & $3.20 \mathrm{~mm}$ & $6.38 \mathrm{~mm}$ \\
Stacked 2-D data & $1.00 \mathrm{~mm}$ & $1.23 \mathrm{~mm}$ \\
Lateral FWHM & & \\
MLSL & $40.9 \mathrm{~dB}$ & $39.2 \mathrm{~dB}$ \\
0.09 mm step & $39.0 \mathrm{~dB}$ & $26.4 \mathrm{~dB}$ \\
0.45 mm step & $15.4 \mathrm{~dB}$ & $8.5 \mathrm{~dB}$ \\
0.99 mm step & $13.5 \mathrm{~dB}$ & $9.4 \mathrm{~dB}$ \\
Stacked 2-D data &
\end{tabular}

${ }^{1} \mathrm{MLSL}=$ main-lobe to side-lobe; FWHM $=$ full-width half max.

simulations and 1.44 and 5.19 for the measured PSF, resulting in a much more uniform PSF. This will allow a more homogeneous image to be obtained regardless of the viewing direction.

\section{Cyst Phantom}

A cyst-mimicking phantom, where the cyst is created with tubes, was scanned at an angle from the elevation direction of the tubes. Fig. 9 shows an image of the cyst with some homogeneous speckle on the left part. The cyst is almost not visible with a $40 \mathrm{~dB}$ dynamic range without elevation focusing and shows an improvement of $5.80 \mathrm{~dB}$ in CNR when applying 3-D SA focusing.

\section{CONCLuSion}

The method shows the ability of calculating the ToF for a spherical sound wave emitted by a linear array trans-

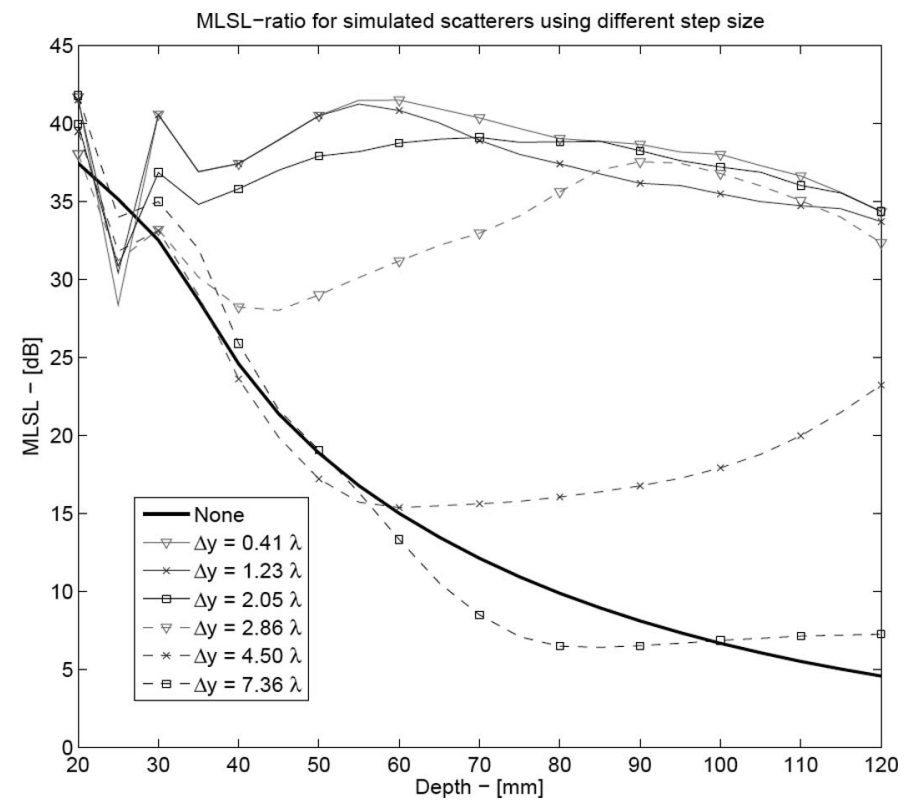

Fig. 7. Main-lobe to side-lobe (MLSL) ratio for simulated scatterers between 20 and $120 \mathrm{~mm}$ of depth. Each line represents a different step size in elevation.

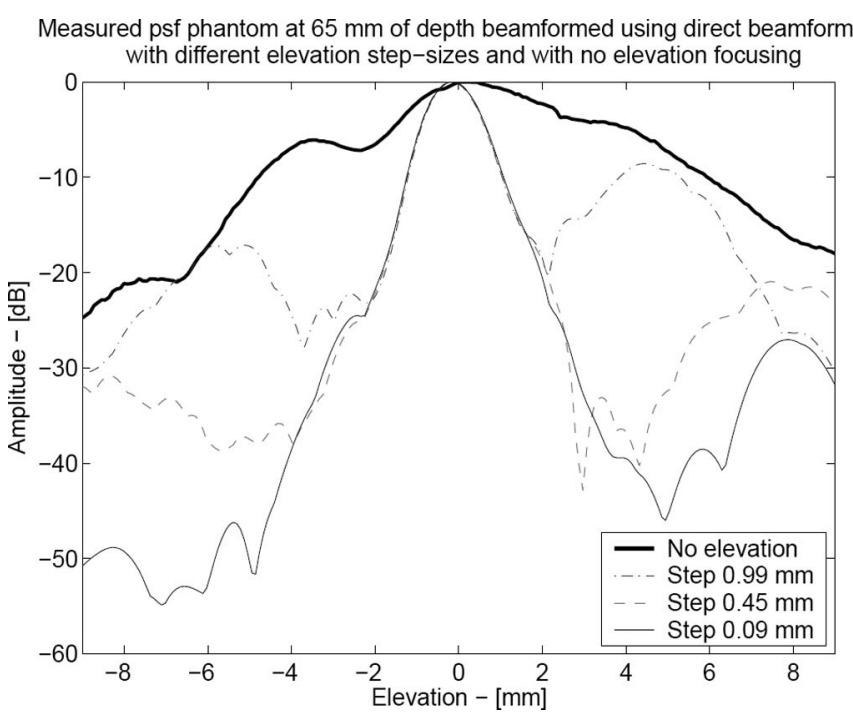

Fig. 8. Projection of the PSF measured on a PSF phantom at $65 \mathrm{~mm}$ of depth for different elevation step sizes.

ducer for any point within the acceptance angles of the VS defined by the transmit VS and the elevation focus VS. A bias of $0.090 \lambda$ and a standard deviation of the error of $0.0054 \lambda$ are found when comparing (2) to the simulated pulse-echo response of a single scatterer placed off-axis. This is an improvement compared with the standard deviation of the 2-step method, which is at $0.0945 \lambda$ for the same scatterer.

The method shows the ability of maintaining a constant $\mathrm{F} \#$ in the elevation direction allowing an almost constant FWHM. For a simulated scatterer at $70 \mathrm{~mm}$ deep, the elevation FWHM is $3.39 \mathrm{~mm}$ with conventional imaging and $1.58 \mathrm{~mm}$ with 3-D SA focusing. The MLSL is $9.79 \mathrm{~dB}$ with conventional imaging and $14.64 \mathrm{~dB}$ with 3-D SA focusing. 


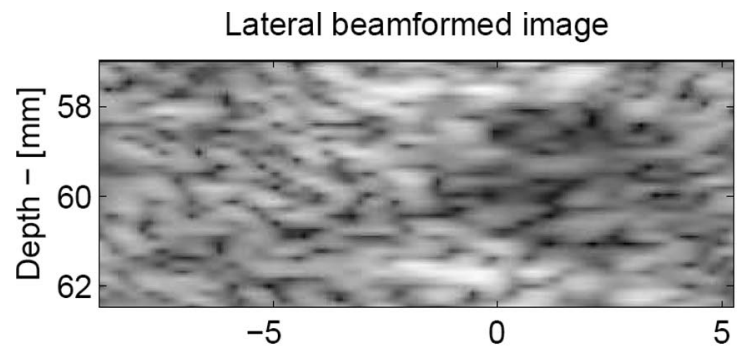

3D SA beamformed image

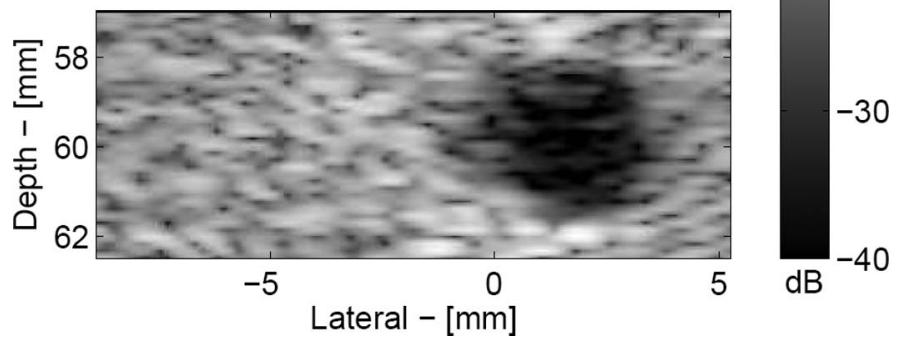

Fig. 9. B-mode image of a cyst phantom with and without synthetic elevation focusing in the lateral-depth plane. The diameter of the cyst is $4 \mathrm{~mm}$.

During a measurement on a PSF phantom containing a small diamond, the FWHM was $6.39 \mathrm{~mm}$ and $1.77 \mathrm{~mm}$ with and without 3-D SA focusing, and the MLSL was $1.09 \mathrm{~dB}$ and $13.08 \mathrm{~dB}$. The measurement showed a greater improvement than the simulations, although the starting position was poorer.

Finally the method was applied to a 3 -D volume scan of a cyst phantom, where the ability to improve the contrast in the conventional imaging plane was evaluated, and an improvement of 5.80 in CNR was found for a $4 \mathrm{~mm}$ waterfilled cyst.

The method allows for a more flexible implementation than previous methods. For a 3-plane visualization of a volume, the method allows a reduction of an order-ofmagnitude in required beamformed points compared with previous methods. Also, the method only requires a single interpolation step and removes the requirement of an intermediate storage of beamformed data.

\section{REFERENCES}

[1] S. W. Smith, H. G. Pavy, and O. T. von Ramm., "High-speed ultrasound volumetric imaging system-Part I: Transducer design and beam steering," IEEE Trans. Ultrason. Ferroelectr. Freq. Control, vol. 38, pp. 100-108, Mar. 1991.

[2] G. R. Lockwood, J. R. Talman, and S. S. Brunke, "Real-time 3-D ultrasound imaging using sparse synthetic aperture beamforming," IEEE Trans. Ultrason. Ferroelectr. Freq. Control, vol. 45, pp. 980988, Jul. 1998.

[3] O. T. von Ramm and S. W. Smith., and HG. Pavy, "High-speed ultrasound volumetric imaging system-Part II: Parallel processing and image display," IEEE Trans. Ultrason. Ferroelectr. Freq. Control, vol. 38, pp. 109-115, Mar. 1991.

[4] C. H. Frazier and W. D. O'Brien, "Synthetic aperture techniques with a virtual source element," IEEE Trans. Ultrason. Ferroelectr. Freq. Control, vol. 45, pp. 196-207, Mar. 1998.

[5] S. I. Nikolov and J. A. Jensen, "3D synthetic aperture imaging using a virtual source element in the elevation plane," in Proc. IEEE Ultrasonics Symp., vol. 2, 2000, pp. 1743-1747.
[6] S. I. Nikolov, "Synthetic aperture tissue and flow ultrasound imaging," Ph.D. dissertation, Ørsted²DTU, Technical Univ. Denmark, Lyngby, Denmark, 2001.

[7] S. I. Nikolov, P. Santén, O. Bjuvsten, and J. A. Jensen, "Parameter study of 3D synthetic aperture post-beamforming procedure," Ultrasonics, vol. 44, pp. e159-e164, Dec. 2006.

[8] J. J. Flaherty, K. R. Erikson, and V. M. Lund, "Synthetic aperture ultrasound imaging systems," U.S. Patent, 3548 642, Dec. 22, 1970.

[9] C. B. Burckhardt, P.-A. Grandchamp, and H. Hoffmann, "An experimental $2 \mathrm{MHz}$ synthetic aperture sonar system intended for medical use," IEEE Trans. Sonics Ultrason., vol. 21, no. 1, pp. 1-6, Jan. 1974.

[10] P. D. Corl, P. M. Grant, and G. S. Kino, "A digital synthetic focus acoustic imaging system for NDE," in Proc. IEEE Ultrasonics Symp., pp. 263-268, 1978.

[11] G. S. Kino, D. Corl, S. Bennett, and K. Peterson, "Real time synthetic aperture imaging system," in Proc. IEEE Ultrasonics Symp., pp. $722-731,1980$.

[12] D. K. Peterson and G. S. Kino, "Real-time digital image reconstruction: A description of imaging hardware and an analysis of quantization errors," IEEE Trans. Sonics Ultrason., vol. 31, no. 4, pp. 337-351, Jul. 1984.

[13] K. Nagai, "A new synthetic-aperture focusing method for ultrasonic Bscan imaging by the fourier transform," IEEE Trans. Sonics Ultrason., vol. SU-32, no. 4, pp. 531-536, 1985.

[14] M. Karaman, P.-C. Li, and M. O'Donnell, "Synthetic aperture imaging for small scale systems," IEEE Trans. Ultrason. Ferroelectr. Freq. Control, vol. 42, pp. 429-442, May 1995.

[15] S. I. Nikolov and J. A. Jensen, "Virtual ultrasound sources in high-resolution ultrasound imaging," in Proc. SPIE-Progress in Biomedical Optics and Imaging, vol. 3, 2002, pp. 395-405.

[16] C. Passmann and H. Ermert, "Adaptive $150 \mathrm{MHz}$ ultasound imaging of the skin and the eye using an optimal combination of short pulse mode and pulse compression mode," in Proc. IEEE Ultrasonics Symp., 1995, pp. 1291-1294.

[17] M. H. Bae, M. K. Jeong, T. K. Song, and Y. B. Ahn, "Experimental study of transmit synthetic focusing combined with receive dynamic focusing in B-mode ultrasound imaging systems," in Proc. IEEE Ultrasonics Symp., 1999, pp. 1261-1264.

[18] F. Gran and J. A. Jensen, "Designing waveforms for temporal encoding using a frequency sampling method," IEEE Trans. Ultrason. Ferroelectr. Freq. Control, vol. 54, no. 10, pp. 2070-2081, Oct. 2007.

[19] K. Gammelmark, "Improving the image quality of synthetic transmit aperture ultrasound images," Ph.D. dissertation, Ørsted ${ }^{2} \mathrm{DTU}$, Technical Univ. Denmark, Lyngby, Denmark, 2004.

[20] G. R. Lockwood and F. S. Foster, "Design of sparse array imaging systems," in Proc. IEEE Ultrasonics Symp., 1995, pp. 1237-1243.

[21] J. A. Jensen, O. Holm, L. J. Jensen, H. Bendsen, S. I. Nikolov, B. G. Tomov, P. Munk, M. Hansen, K. Salomonsen, J. Hansen, K. Gormsen, H. M. Pedersen, and K. L. Gammelmark, "Ultrasound research scanner for real-time synthetic aperture image acquisition," IEEE Trans. Ultrason. Ferroelectr. Freq. Control, vol. 52, no. 5, pp. 881-891, May 2005.

[22] F. Gran and J. A. Jensen, "Designing waveforms for temporal encoding using a frequency sampling method," IEEE Trans. Ultrason. Ferroelectr. Freq. Control, vol. 54, no. 10, pp. 2070-2081, 2007.

[23] Y. Takeuchi, "Chirped excitation for $<-100 \mathrm{~dB}$ time sidelobe echo sounding," in Proc. IEEE Ultrasonics Symp., 1995, pp. 1309-1314.

[24] R. Y. Chiao and L. J. Thomas, "Synthetic transmit aperture using orthogonal Golay coded excitation," in Proc. IEEE Ultrasonics Symp., 2000, pp. 1469-1472.

[25] J. Shen and E. S. Ebbini, "A new coded-excitation ultrasound imaging system-Part I: Basic principles," IEEE Trans. Ultrason. Ferroelectr. Freq. Control, vol. 43, pp. 131-140, Jan. 1996.

[26] J. A. Jensen and N. B. Svendsen., "Calculation of pressure fields from arbitrarily shaped, apodized, and excited ultrasound transducers," IEEE Trans. Ultrason. Ferroelectr. Freq. Control, vol. 39, pp. 262-267, Mar. 1992.

[27] J. A. Jensen, "Field: A program for simulating ultrasound systems," in Med. Biol. Eng. Comp., 10th Nordic-Baltic Conf. Biomedical Imaging, 1996b, vol. 4, suppl. 1, part 1:351-353.

[28] F. N. Ucar and M. Karaman, "Beam space processing for lowcost scanners," in Proc. IEEE Ultrasonics Symp., 1996, pp. 13491352. 


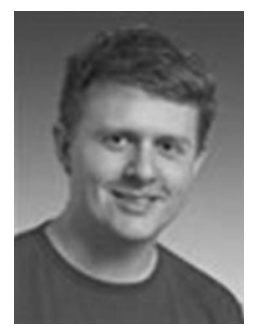

Henrik Andresen earned his M.Sc. degree in electrical engineering in 2006 from the Technical University of Denmark, Lyngby, Denmark. He is currently working toward the Ph.D. degree in electrical engineering at the same university. His research interests are focused on applying synthetic aperture focusing techniques for improving 3-D ultrasound.

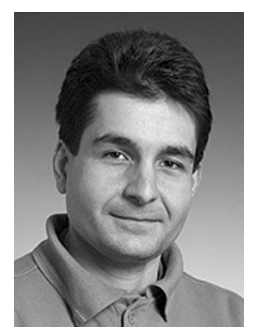

Svetoslav I. Nikolov received M.Sc. degree in electrical engineering and MBA degree in international business relations from the Technical University of Sofia, Sofia, Bulgaria, in 1996 and 1997, respectively. In 2001, he got a Ph.D. degree from the Technical University of Denmark, Lyngby, Denmark. His dissertation explored approaches for synthetic aperture tissue and flow imaging and possibilities for real-time 3-D imaging.

After completing his doctoral work, he stayed on at Ørsted Technical University of Denmark, first as an assistant and later as an associate professor, where he taught digital design, software development, and digital signal processing. Presently, Dr. Nikolov works at BK Medical AS in the R\&D Department and teaches advanced signal processing at the Technical University of Denmark. His current research interests are focused on real-time systems and signal processing and methods for high-resolution tissue and flow imaging.

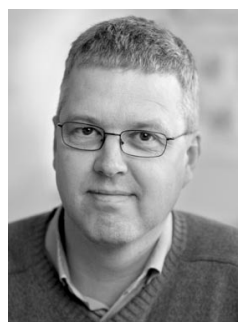

Jorgen Arendt Jensen earned his M.Sc. degree in electrical engineering in 1985 and the Ph.D. degree in 1989, both from the Technical University of Denmark, Lyngby, Denmark. He received the Dr. Techn. degree from the same university in 1996. He has published more than 160 journal and conference papers on signal processing and medical ultrasound, and his book Estimation of Blood Velocities Using Ultrasound was published by Cambridge University Press in 1996. He is also developer of the Field II simulation program. He has been a visiting scientist at Duke University, Stanford University, and the University of Illinois at Urbana-Champaign. He is currently full professor of biomedical signal processing at the Technical University of Denmark in the Department of Electrical Engineering and head of the Center for Fast Ultrasound Imaging and the Group for Biomedical Engineering. He is also adjunct full professor with the Faculty of Health Sciences at the University of Copenhagen. He has given courses on blood velocity estimation at both Duke University and University of Illinois and teaches biomedical signal processing and medical imaging at the Technical University of Denmark. He has given several short courses on simulation, synthetic aperture imaging, and flow estimation at international scientific conferences. He has received several awards for his research. He is also the co-organizer of B.Sc./M.Sc. education in biomedical engineering offered jointly by the Technical University of Denmark and the University of Copenhagen. His research is centered on simulation of ultrasound imaging, synthetic aperture imaging, vector blood flow estimation, and construction of ultrasound research systems. 